\title{
ABELIAN QUOTIENTS ARISING FROM EXTRIANGULATED CATEGORIES VIA MORPHISM CATEGORIES
}

\author{
ZENGQIANG LIN
}

\begin{abstract}
We investigate abelian quotients arising from extriangulated categories via morphism categories, which is a unified treatment for both exact categories and triangulated categories. Let $(\mathcal{C}, \mathbb{E}, \mathfrak{s})$ be an extriangulated category with enough projectives $\mathcal{P}$ and $\mathcal{M}$ be a full subcategory of $\mathcal{C}$ containing $\mathcal{P}$. We show that certain quotient category of $\mathfrak{s}-\operatorname{def}(\mathcal{M})$, the category of $\mathfrak{s}$-deflations $f: M_{1} \rightarrow M_{2}$ with $M_{1}, M_{2} \in \mathcal{M}$, is abelian. Our main theorem has two applications. If $\mathcal{M}=\mathcal{C}$, we obtain that certain ideal quotient category $\mathfrak{s}$-tri $(\mathcal{C}) / \mathcal{R}_{2}$ is equivalent to the category of finitely presented modules mod- $\mathcal{C} /[\mathcal{P}]$, where $\mathfrak{s}$-tri $(\mathcal{C})$ is the category of all $\mathfrak{s}$-triangles. If $\mathcal{M}$ is a rigid subcategory, we show that $\mathcal{M}_{L} /[\mathcal{M}] \cong \bmod -(\mathcal{M} /[\mathcal{P}])$ and $\mathcal{M}_{L} /[\Omega \mathcal{M}] \cong\left(\bmod -(\mathcal{M} /[\mathcal{P}])^{\mathrm{op}}\right)^{\mathrm{op}}$, where $\mathcal{M}_{L}$ (resp. $\left.\Omega \mathcal{M}\right)$ is the full subcategory of $\mathcal{C}$ of objects $X$ admitting an $\mathfrak{s}-$ triangle $X \longrightarrow M_{1} \longrightarrow M_{2}--\rightarrow($ resp. $X \longrightarrow P \longrightarrow M-->$ ) with $M_{1}, M_{2} \in \mathcal{M}$ (resp. $\quad M \in \mathcal{M}$ and $P \in \mathcal{P}$ ). In particular, we have $\mathcal{C} /[\mathcal{M}] \cong \bmod -(\mathcal{M} /[\mathcal{P}])$ and $\mathcal{C} /[\Omega \mathcal{M}] \cong\left(\bmod -(\mathcal{M} /[\mathcal{P}])^{\text {op }}\right)^{\text {op }}$ provided that $\mathcal{M}$ is a cluster-tilting subcategory.
\end{abstract}

\section{INTRODUCTION}

In representation theory, there are a few quotient categories admitting natural abelian structures. For both triangulated categories and exact categories, clustertilting subcategories provide a way to construct abelian quotient categories. Let $\mathcal{C}$ be a triangulated category and $\mathcal{T}$ be a cluster-tilting subcategory of $\mathcal{C}$, then the quotient $\mathcal{C} /[\mathcal{T}]$ is abelian; related works see $[1,8,9$. The version of exact categories see 2]. Submodule categories provide another way to construct abelian quotient categories. Certain quotients of submodule categories are realized as categories of finitely presented modules over stable Auslander algebras [16, 3]. More generally, some quotients of categories of short exact sequences in exact categories are abelian, related works see [4, 10. For triangulated version, certain quotients of categories of triangles are abelian [14.

Recently, Nakaoka and Palu introduced the notion of extriangulated categories 15, which is a simultaneous generalization of exact categories and triangulated categories. They pointed out that the notion is a convenient setup for writing down proofs which apply to both exact categories and triangulated categories. For recent developments on extriangulated categories we refer to [6, 11, 12, 13, 17, etc.

In this paper, we focus our attention onto the abelian quotients arising from extriangulated categories via morphism categories, which is a unified treatment of

2010 Mathematics Subject Classification. 18E30.

Key words and phrases. extriangulated categories; abelian categories.

This work was supported by the national natural science foundation of China (Grants No. 11871014 and No. 11871259). 
abelian quotients for both exact categories and triangulated categories. Our approach to abelian quotients is based on identifying quotients of morphism categories as certain module categories.

Let $(\mathcal{C}, \mathbb{E}, \mathfrak{s})$ be an extriangulated category and $\mathcal{M}$ be a full subcategory of $\mathcal{C}$. We denote by $\operatorname{Mor}(\mathcal{M})$ the morphism category of $\mathcal{M}$ and by $\mathfrak{s}-\operatorname{def}(\mathcal{M})(\operatorname{resp} . \mathfrak{s}-\inf (\mathcal{M}))$ the full subcategory of $\operatorname{Mor}(\mathcal{M})$ consisting of $\mathfrak{s}$-deflations (resp. $\mathfrak{s}$-inflations). The full subcategory of $\mathfrak{s}-\operatorname{def}(\mathcal{M})$ consisting of split epimorphisms (resp. split monomorphisms) is denoted by s-epi $(\mathcal{M})(\operatorname{resp} . \operatorname{s-mono}(\mathcal{M}))$. We denote by $\operatorname{sp-epi}(\mathcal{M})$ (resp. si-mono $(\mathcal{M}))$ the full subcategory of $\mathfrak{s}-\operatorname{def}(\mathcal{M})$ consisting of $(M \stackrel{1}{\rightarrow} M) \oplus\left(P \rightarrow M^{\prime}\right)$ (resp. $\left.(M \stackrel{1}{\rightarrow} M) \oplus\left(M^{\prime} \rightarrow I\right)\right)$ with $P \in \mathcal{P}$ (resp. $\left.I \in \mathcal{I}\right)$.

Our main theorem is the following (Theorem 3.2 ), which generalizes $[10$, Theorem $3.9]$.

Theorem 1.1. Let $\mathcal{C}$ be an extriangulated category and $\mathcal{M}$ be a full subcategory of $\mathcal{C}$.

(1) If $\mathcal{C}$ has enough projectives $\mathcal{P}$ and $\mathcal{M}$ contains $\mathcal{P}$, then $\mathfrak{s}-\operatorname{def}(\mathcal{M}) /[\operatorname{s-epi}(\mathcal{M})] \cong$ $\bmod -\mathcal{M} /[\mathcal{P}]$ and $\mathfrak{s}-\operatorname{def}(\mathcal{M}) /[\operatorname{sp}-\operatorname{epi}(\mathcal{M})] \cong\left(\bmod -(\mathcal{M} /[\mathcal{P}])^{\mathrm{op}}\right)^{\mathrm{op}}$.

(2) If $\mathcal{C}$ has enough injectives $\mathcal{I}$ and $\mathcal{M}$ contains $\mathcal{I}$, then $\mathfrak{s}-\inf (\mathcal{M}) /[\operatorname{s}-\operatorname{mono}(\mathcal{M})] \cong$ $\left(\bmod -(\mathcal{M} /[\mathcal{I}])^{\text {op }}\right)^{\text {op }}$ and $\mathfrak{s}-\inf (\mathcal{M}) /[\operatorname{si}-\operatorname{mono}(\mathcal{M})] \cong \bmod -\mathcal{M} /[\mathcal{I}]$.

Theorem 1.1 has two interesting applications. We will investigate two special cases when $\mathcal{M}=\mathcal{C}$ and when $\mathcal{M}$ is rigid, that is, $\mathbb{E}\left(M, M^{\prime}\right)=0$ for any $M, M^{\prime} \in \mathcal{M}$.

For the first case, we denote by $\mathfrak{s}$-tri $(\mathcal{C})$ the category of all $\mathfrak{s}$-triangles, where the objects are the $\mathfrak{s}$-triangles $X_{\bullet}=\left(X_{1} \stackrel{f_{1}}{\longrightarrow} X_{2} \stackrel{f_{2}}{\longrightarrow} X_{3}-\stackrel{\delta}{-}>\right)$ and the morphisms from $X_{\bullet}$ to $Y_{\bullet}$ are the triples $\varphi_{\bullet}=\left(\varphi_{1}, \varphi_{2}, \varphi_{3}\right)$ such that the following diagram

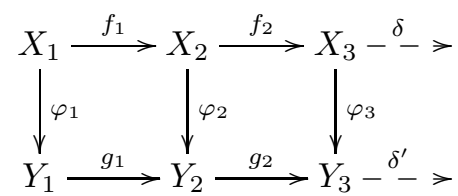

is a morphism of $\mathfrak{s}$-triangles. Let $X_{\bullet}$ and $Y_{\bullet}$ be two $\mathfrak{s}$-triangles, we denote by $\mathcal{R}_{2}\left(X_{\bullet}, Y_{\bullet}\right)$ the class of morphisms $\varphi_{\bullet}: X_{\bullet} \rightarrow Y_{\bullet}$ such that $\varphi_{3}$ factors through $g_{2}$. It is easy to see that $\mathcal{R}_{2}$ is an ideal of $\mathfrak{s}$-tri $(\mathcal{C})$, moreover, the following three quotient categories $\mathfrak{s}$-tri $(\mathcal{C}) / \mathcal{R}_{2}, \mathfrak{s}-\operatorname{def}(\mathcal{C}) /[\operatorname{s-epi}(\mathcal{C})]$ and $\mathfrak{s}$-inf $(\mathcal{C}) /[\operatorname{s-mono}(\mathcal{C})]$ are equivalent.

Given an $\mathfrak{s}$-triangle $\delta=\left(X_{1} \stackrel{f_{1}}{\longrightarrow} X_{2} \stackrel{f_{2}}{\longrightarrow} X_{3}-\stackrel{\rho}{-} \rightarrow\right)$, we define the contravariant defect $\delta^{*}$ and the covariant defect $\delta_{*}$ by the following exact sequence of functors

$$
\begin{aligned}
& \mathcal{C}\left(-, X_{1}\right) \stackrel{\mathcal{C}\left(-, f_{1}\right)}{\longrightarrow} \mathcal{C}\left(-, X_{2}\right) \stackrel{\mathcal{C}\left(-, f_{2}\right)}{\longrightarrow} \mathcal{C}\left(-, X_{3}\right) \rightarrow \delta^{*} \rightarrow 0, \\
& \mathcal{C}\left(X_{3},-\right) \stackrel{\mathcal{C}\left(f_{2},-\right)}{\longrightarrow} \mathcal{C}\left(X_{2},-\right) \stackrel{\mathcal{C}\left(f_{1},-\right)}{\longrightarrow} \mathcal{C}\left(X_{1},-\right) \rightarrow \delta_{*} \rightarrow 0 .
\end{aligned}
$$

Our first application of Theorem 1.1 is the following (Theorem 4.1, Proposition 4.3 and Theorem 4.8), which generalizes [10, Theorem 4.1, Theorem 4.8, Theorem 5.1].

Theorem 1.2. Let $\mathcal{C}$ be an extriangulated category.

(1) The quotient $\mathfrak{s}$-tri $(\mathcal{C}) / \mathcal{R}_{2}$ is abelian.

(2) If $\mathcal{C}$ has enough projectives $\mathcal{P}$, then we have the following equivalence

$$
F: \mathfrak{s} \text {-tri }(\mathcal{C}) / \mathcal{R}_{2} \cong \bmod -\mathcal{C} /[\mathcal{P}], \delta \mapsto \delta^{*}
$$


(3) If $\mathcal{C}$ has enough injectives $\mathcal{I}$, then we have the following equivalence

$$
G: \mathfrak{s}-\operatorname{tri}(\mathcal{C}) / \mathcal{R}_{2} \cong\left(\bmod -(\mathcal{C} /[\mathcal{I}])^{\text {op }}\right)^{\text {op }}, \delta \mapsto \delta_{*} .
$$

We point out that the abelian quotient $\mathfrak{s}-\operatorname{tri}(\mathcal{C}) / \mathcal{R}_{2}$ admits nice properties. We describe the projectives, injectives and simple objects in $\mathfrak{s}$-tri $(\mathcal{C}) / \mathcal{R}_{2} ;$ see Proposition 4.4 and Proposition 4.6. In particular, if $\mathcal{C}$ has enough projectives $\mathcal{P}$ and enough injectives $\mathcal{I}$, then there is a duality between $\bmod -\mathcal{C} /[\mathcal{P}]$ and $\bmod -(\mathcal{C} /[\mathcal{I}])^{\text {op }}$, which is used to derive Auslander-Reiten duality and defect formula for extriangulated categories; see Proposition 4.9.

For describing the second application, we first give some notations. Let $\mathcal{C}^{\prime}$ and $\mathcal{C}^{\prime \prime}$ be two full subcategories of $\mathcal{C}$. We denote by Cocone $\left(\mathcal{C}^{\prime}, \mathcal{C}^{\prime \prime}\right)\left(\operatorname{resp}\right.$. Cone $\left.\left(\mathcal{C}^{\prime}, \mathcal{C}^{\prime \prime}\right)\right)$ the subcategory of objects $X$ admitting an s-triangle $X \longrightarrow C^{\prime} \longrightarrow C^{\prime \prime}--\rightarrow$ (resp. $C^{\prime} \longrightarrow C^{\prime \prime} \longrightarrow X--\rightarrow$ ) with $C^{\prime} \in \mathcal{C}^{\prime}$ and $C^{\prime \prime} \in \mathcal{C}^{\prime \prime}$.

Let $\mathcal{M}$ be a rigid subcategory of $\mathcal{C}$. For convenience we let $\mathcal{M}_{L}=\operatorname{Cocone}(\mathcal{M}, \mathcal{M})$ and $\mathcal{M}_{R}=\operatorname{Cone}(\mathcal{M}, \mathcal{M})$. If $\mathcal{C}$ has enough projectives $\mathcal{P}$ and enough injectives $\mathcal{I}$, then we let $\Omega \mathcal{M}=\operatorname{Cocone}(\mathcal{P}, \mathcal{M})$ and $\Sigma \mathcal{M}=\operatorname{Cone}(\mathcal{M}, \mathcal{I})$. It turns out that the quotient categories $\mathfrak{s}-\operatorname{def}(\mathcal{M}) /[\operatorname{s-epi}(\mathcal{M})]$ and $\mathfrak{s}-\operatorname{def}(\mathcal{M}) /[\operatorname{sp}-\operatorname{epi}(\mathcal{M})]$ can be realized as subquotient categories of $\mathcal{C}$.

Our second application of Theorem 1.1 is the following (Theorem 5.4), which generalizes [2, Theorem 3.2, Theorem 3.4] and [7, Proposition 6.2].

Theorem 1.3. Let $\mathcal{C}$ be an extriangulated category and $\mathcal{M}$ be a rigid subcategory of $\mathcal{C}$.

(1) If $\mathcal{C}$ has enough projectives $\mathcal{P}$ and $\mathcal{M}$ contains $\mathcal{P}$, then $\mathcal{M}_{L} /[\mathcal{M}] \cong \bmod -(\mathcal{M} /[\mathcal{P}])$ and $\mathcal{M}_{L} /[\Omega \mathcal{M}] \cong\left(\bmod -(\mathcal{M} /[\mathcal{P}])^{\mathrm{op}}\right)^{\mathrm{op}}$

(2) If $\mathcal{C}$ has enough injectives $\mathcal{I}$ and $\mathcal{M}$ contains $\mathcal{I}$, then $\mathcal{M}_{R} /[\mathcal{M}] \cong\left(\bmod -(\mathcal{M} /[\mathcal{I}])^{\mathrm{op}}\right)^{\mathrm{op}}$ and $\mathcal{M}_{R} /[\Sigma \mathcal{M}] \cong \bmod -(\mathcal{M} /[\mathcal{I}])$.

In particular, if $\mathcal{M}$ is a cluster tilting subcategory of $\mathcal{C}$, then $\mathcal{M}_{L}=\mathcal{M}_{R}=\mathcal{C}$. Thus we have the following result (Corollary 5.7).

Corollary 1.4. Let $\mathcal{C}$ be an extriangulated category with enough projectives $\mathcal{P}$ and enough injectives $\mathcal{I}$. If $\mathcal{M}$ is a cluster tilting subcategory of $\mathcal{C}$, then

(1) $\mathcal{C} /[\mathcal{M}] \cong \bmod -(\mathcal{M} /[\mathcal{P}]) \cong\left(\bmod -(\mathcal{M} /[\mathcal{I}])^{\mathrm{op}}\right)^{\mathrm{op}}$.

(2) $\mathcal{C} /[\Omega \mathcal{M}] \cong\left(\bmod -(\mathcal{M} /[\mathcal{P}])^{\mathrm{op}}\right)^{\mathrm{op}}$.

(3) $\mathcal{C} /[\Sigma \mathcal{M}] \cong \bmod -(\mathcal{M} /[\mathcal{I}])$.

This paper is organized as follows. In Section 2 we make some preliminaries on morphism categories and extriangulated categories. In Section 3 we prove Theorem 1.1. In Section 4 we provide the first application. In Section 5 we provide the second application.

\section{DEFINITIONS AND PRELIMINARIES}

In this section, we first give some facts on morphism categories, then recall the definitions and basic properties on extriangulated categories from [15], 11] and [6].

2.1. Morphism categories. Let $\mathcal{C}$ be an additive category. The morphism category of $\mathcal{C}$ is the category $\operatorname{Mor}(\mathcal{C})$ defined by the following data. The objects of $\operatorname{Mor}(\mathcal{C})$ are all the morphisms $f: X \rightarrow Y$ in $\mathcal{C}$. The morphisms from $f: X \rightarrow Y$ to $f^{\prime}: X^{\prime} \rightarrow Y^{\prime}$ are pairs $(a, b)$ where $a: X \rightarrow X^{\prime}$ and $b: Y \rightarrow Y^{\prime}$ such that $b f=f^{\prime} a$. The composition of morphisms is componentwise. For two objects $f: X \rightarrow Y$ 
and $f^{\prime}: X^{\prime} \rightarrow Y^{\prime}$ in $\operatorname{Mor}(\mathcal{C})$, we define $\mathcal{R}\left(f, f^{\prime}\right)$ (resp. $\left.\mathcal{R}^{\prime}\left(f, f^{\prime}\right)\right)$ to be the set of morphisms $(a, b)$ such that there is some morphism $p: Y \rightarrow X^{\prime}$ such that $f^{\prime} p=b$ $\left(\right.$ resp. $p f=a$ ). Then $\mathcal{R}$ and $\mathcal{R}^{\prime}$ are ideals of $\operatorname{Mor}(\mathcal{C})$. We denote by s-epi $(\mathcal{C})$ (resp. s-mono $(\mathcal{C})$ ) the full subcategory of $\operatorname{Mor}(\mathcal{C})$ consisting of split epimorphisms (resp. split monomorphisms).

Recall that a right $\mathcal{C}$-module is a contravariantly additive functor $F: \mathcal{C} \rightarrow A b$, where $A b$ is the category of abelian groups. A $\mathcal{C}$-module $F$ is called finitely presented if there exists an exact sequence $\mathcal{C}(-, X) \rightarrow \mathcal{C}(-, Y) \rightarrow F \rightarrow 0$. We denote by mod$\mathcal{C}$ the category of finitely presented $\mathcal{C}$-modules, and by proj- $\mathcal{C}$ (resp. inj- $\mathcal{C}$ ) the full subcategory of mod-C consisting of projectives (resp. injectives).

The following result was proved in [10, Lemma 3.1] and [10, Proposition 3.3].

Lemma 2.1. Let $\mathcal{C}$ be an additive category, then

(1) $\operatorname{Mor}(\mathcal{C}) / \mathcal{R} \cong \operatorname{Mor}(\mathcal{C}) /[\operatorname{s-epi}(\mathcal{C})] \cong \bmod -\mathcal{C}$.

(2) $\operatorname{Mor}(\mathcal{C}) / \mathcal{R}^{\prime} \cong \operatorname{Mor}(\mathcal{C}) /[\operatorname{s-mono}(\mathcal{C})] \cong\left(\bmod -\mathcal{C}^{\mathrm{op}}\right)^{\mathrm{op}}$.

2.2. Extriangulated categories. Let $\mathcal{C}$ be an additive category equipped with an additive bifunctor $\mathbb{E}: \mathcal{C}^{\text {op }} \times \mathcal{C} \rightarrow A b$. For any pair of objects $A, C \in \mathcal{C}$, an object $\delta \in \mathbb{E}(C, A)$ is called an $\mathbb{E}$-extension. For any morphism $a \in \mathcal{C}\left(A, A^{\prime}\right)$ and $c \in \mathcal{C}\left(C^{\prime}, C\right)$, we denote the $\mathbb{E}$-extension $\mathbb{E}(C, a)(\delta) \in \mathbb{E}\left(C, A^{\prime}\right)$ by $a_{*} \delta$ and denote the $\mathbb{E}$-extension $\mathbb{E}(c, A)(\delta) \in \mathbb{E}\left(C^{\prime}, A\right)$ by $c^{*} \delta$. Let $\delta \in \mathbb{E}(C, A)$ and $\delta^{\prime} \in \mathbb{E}\left(C^{\prime}, A^{\prime}\right)$ be two $\mathbb{E}$-extensions. A morphism $(a, c): \delta \rightarrow \delta^{\prime}$ of $\mathbb{E}$-extensions is a pair of morphisms $a \in \mathcal{C}\left(A, A^{\prime}\right)$ and $c \in \mathcal{C}\left(C, C^{\prime}\right)$ such that $a_{*} \delta=c^{*} \delta^{\prime}$.

Let $A, C \in \mathcal{C}$ be any pair of objects. Two sequences of morphisms in $\mathcal{C}$

$$
A \stackrel{x}{\rightarrow} B \stackrel{y}{\rightarrow} C \text { and } A \stackrel{x^{\prime}}{\rightarrow} B^{\prime} \stackrel{y^{\prime}}{\rightarrow} C
$$

are equivalent if there exists an isomorphism $b \in \mathcal{C}\left(B, B^{\prime}\right)$ such that the following diagram is commutative.

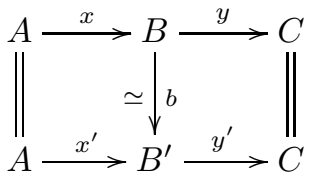

We denote the equivalence class of $A \stackrel{x}{\rightarrow} B \stackrel{y}{\rightarrow} C$ by $[A \stackrel{x}{\rightarrow} B \stackrel{y}{\rightarrow} C]$.

Definition 2.2. Let $\mathbb{E}: \mathcal{C}^{\mathrm{op}} \times \mathcal{C} \rightarrow A b$ be an additive bifunctor. A correspondence $\mathfrak{s}$ is called a realization of $\mathbb{E}$ if it associates an equivalence class $\mathfrak{s}(\delta)=[A \stackrel{x}{\rightarrow} B \stackrel{y}{\rightarrow} C]$ to any $\mathbb{E}$-extension $\delta \in \mathbb{E}(C, A)$ and associates a commutative diagram

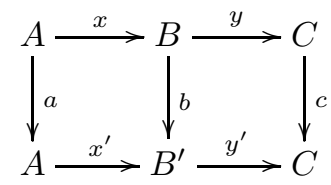

to any morphism $(a, c): \delta \rightarrow \delta^{\prime}$ of $\mathbb{E}$-extensions, where $\mathfrak{s}(\delta)=[A \stackrel{x}{\rightarrow} B \stackrel{y}{\rightarrow} C]$ and $\mathfrak{s}\left(\delta^{\prime}\right)=\left[A^{\prime} \stackrel{x^{\prime}}{\rightarrow} B^{\prime} \stackrel{y^{\prime}}{\rightarrow} C^{\prime}\right]$. In the above situation, we say the sequence $A \stackrel{x}{\rightarrow} B \stackrel{y}{\rightarrow} C$ realizes $\delta$ and the triple $(a, b, c)$ realizes $(a, c)$.

Definition 2.3. A realization $\mathfrak{s}$ of $\mathbb{E}$ is said to be additive if it satisfies the following two conditions. 

$C]$.

(1) Assume that $0 \in \mathbb{E}(C, A)$ is the zero element, then $\mathfrak{s}(0)=\left[A \stackrel{\left(\begin{array}{l}1 \\ 0\end{array}\right)}{\longrightarrow} A \oplus C \stackrel{(0,1)}{\longrightarrow}\right.$

(2) Assume that $\mathfrak{s}(\delta)=[A \stackrel{x}{\rightarrow} B \stackrel{y}{\rightarrow} C]$ and $\mathfrak{s}\left(\delta^{\prime}\right)=\left[A^{\prime} \stackrel{x^{\prime}}{\rightarrow} B^{\prime} \stackrel{y^{\prime}}{\rightarrow} C^{\prime}\right]$, then $\mathfrak{s}\left(\delta \oplus \delta^{\prime}\right)=\left[A \oplus A^{\prime} \stackrel{x \oplus x^{\prime}}{\longrightarrow} B \oplus B^{\prime} \stackrel{y \oplus y^{\prime}}{\longrightarrow} C \oplus C^{\prime}\right]$, where $\delta \oplus \delta^{\prime} \in \mathbb{E}\left(C \oplus C^{\prime}, A \oplus A^{\prime}\right)$ is the element corresponding to $\left(\delta, 0,0, \delta^{\prime}\right)$ under the isomorphism $\mathbb{E}\left(C \oplus C^{\prime}, A \oplus A^{\prime}\right) \cong$ $\mathbb{E}(C, A) \oplus \mathbb{E}\left(C, A^{\prime}\right) \oplus \mathbb{E}\left(C^{\prime}, A\right) \oplus \mathbb{E}\left(C^{\prime}, A^{\prime}\right)$.

Let $\mathfrak{s}$ be an additive realization of $\mathbb{E}$. If $\mathfrak{s}(\delta)=[A \stackrel{x}{\rightarrow} B \stackrel{y}{\rightarrow} C]$, then the sequence $A \stackrel{x}{\rightarrow} B \stackrel{y}{\rightarrow} C$ is called an $\mathfrak{s}$-conflation, the morphism $x$ is called an $\mathfrak{s}$-inflation and $y$ is called an $\mathfrak{s}$-deflation. In this case, we say $A \stackrel{x}{\longrightarrow} B \stackrel{y}{\longrightarrow} C-\stackrel{\delta}{\longrightarrow}$ is an $\mathfrak{s}$-triangle. Let $A \stackrel{x}{\longrightarrow} B \stackrel{y}{\longrightarrow} C-\stackrel{\delta}{\longrightarrow}$ and $A^{\prime} \stackrel{x^{\prime}}{\longrightarrow} B^{\prime} \stackrel{y^{\prime}}{\longrightarrow} C^{\prime}-\delta^{\prime} \rightarrow$ be any pair of $\mathfrak{s}$-triangles. Let $(a, c): \delta \rightarrow \delta^{\prime}$ be a morphism of $\mathbb{E}$-extensions. If a triple $(a, b, c)$ realizes $(a, c)$, then we say $(a, b, c)$ is a morphism of $\mathfrak{s}$-triangles.

Definition 2.4. ([15, Definition 2.12]) A triple $(\mathcal{C}, \mathbb{E}, \mathfrak{s})$ is an extriangulated category if the following conditions are satisfied.

$(\mathrm{ET} 1) \mathbb{E}: \mathcal{C}^{\mathrm{op}} \times \mathcal{C} \rightarrow A b$ is an additive bifunctor.

$(\mathrm{ET} 2) \mathfrak{s}$ is an additive realization of $\mathbb{E}$.

(ET3) Each commutative diagram

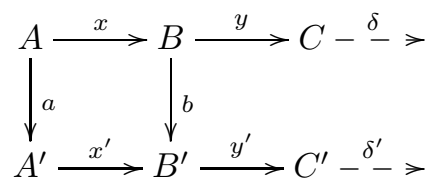

whose rows are $\mathfrak{s}$-triangles can be completed to a morphism of $\mathfrak{s}$-triangles.

(ET3) ${ }^{\text {op }}$ Each commutative diagram

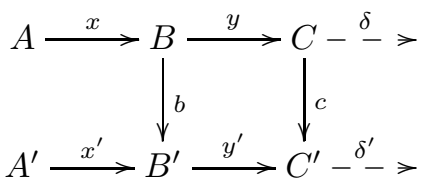

whose rows are $\mathfrak{s}$-triangles can be completed to a morphism of $\mathfrak{s}$-triangles.

(ET4) Let $A \stackrel{f}{\longrightarrow} B \stackrel{f^{\prime}}{\longrightarrow} D-\stackrel{\delta}{\longrightarrow}$ and $B \stackrel{g}{\longrightarrow} C \stackrel{g^{\prime}}{\longrightarrow} F-\stackrel{\delta^{\prime}}{\longrightarrow}$ be $\mathfrak{s}-$ triangles. There exists a commutative diagram

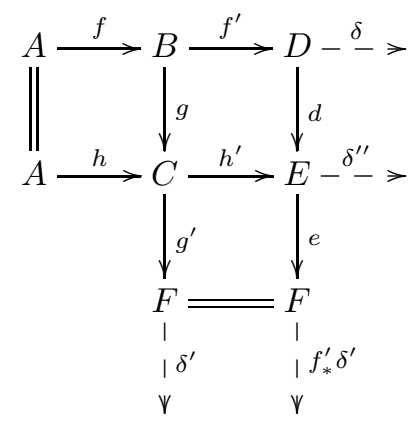


such that the second row and the third column are $\mathfrak{s}$-triangles, moreover, $\delta=d^{*} \delta^{\prime \prime}$ and $f_{*} \delta^{\prime \prime}=e^{*} \delta^{\prime}$.

$(\mathrm{ET} 4)^{\mathrm{op}}$ Let $D \stackrel{f^{\prime}}{\longrightarrow} A \stackrel{f}{\longrightarrow} B-\stackrel{\delta}{\longrightarrow}>$ and $F \stackrel{g^{\prime}}{\longrightarrow} B \stackrel{g}{\longrightarrow} C-\stackrel{\delta^{\prime}}{\longrightarrow}$ be $\mathfrak{s}-$ triangles. There exists a commutative diagram

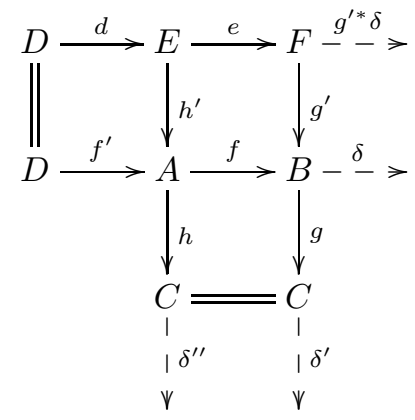

such that the first row and the second column are $\mathfrak{s}$-triangles, moreover, $\delta^{\prime}=e_{*} \delta^{\prime \prime}$ and $d_{*} \delta=g^{*} \delta^{\prime \prime}$.

Definition 2.5. Let $(\mathcal{C}, \mathbb{E}, \mathfrak{s})$ be an extriangulated category.

(1) An object $P \in \mathcal{C}$ is called projective if for any $\mathfrak{s}$-deflation $y: B \rightarrow C$ and any morphism $c: P \rightarrow C$, there exists a morphism $b: P \rightarrow B$ such that $y b=c$. The full subcategory of projectives is denoted by $\mathcal{P}$.

(2) We say that $\mathcal{C}$ has enough projectives if for any object $C \in \mathcal{C}$ there exists an s-triangle $A \stackrel{x}{\longrightarrow} P \stackrel{y}{\longrightarrow} C-\stackrel{\delta}{\longrightarrow}$ with $P \in \mathcal{P}$.

Example 2.6. [15, Example 3.26] (1) Let $\mathcal{C}$ be an exact category, then $\mathcal{C}$ is an extriangulated category with $\mathbb{E}(-,-)=\operatorname{Ext}_{\mathcal{C}}^{1}(-,-)$. In particular, if $\mathcal{C}$ is an exact category with enough projectives, then $\mathcal{C}$ is an extriangulated category with enough projectives.

(2) Let $\mathcal{C}$ be a triangulated category with shift functor [1], then $\mathcal{C}$ is an extriangulated category with $\mathbb{E}(-,-)=\mathcal{C}(-,-[1])$. Moreover, $\mathcal{C}$ has enough projectives. In this case, $\mathcal{P}$ consists of zero objects.

The following lemmas will be used frequently later.

Lemma 2.7. (15, Corollary 3.5]) Let $\mathcal{C}$ be an extriangulated category. Assume that the diagram

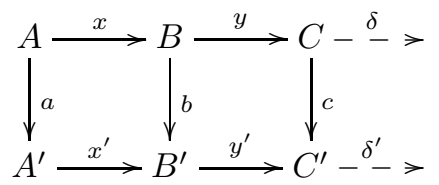

is a morphism of $\mathfrak{s}$-triangles. Then the following statements are equivalent.

(1) a factors through $x$.

(2) $a_{*} \delta=c^{*} \delta^{\prime}=0$.

(3) c factors through $y^{\prime}$.

Lemma 2.8. (11, Proposition 1.20]) Let $\mathcal{C}$ be an extriangulated category. Assume that $A \stackrel{x}{\longrightarrow} B \stackrel{y}{\longrightarrow} C-\stackrel{\delta}{-}$ is an $\mathfrak{s - t r i a n g l e , ~} f: A \rightarrow D$ is a morphism and 
$D \stackrel{d}{\longrightarrow} E \stackrel{e}{\longrightarrow} C \stackrel{f_{*} \delta}{-} \rightarrow$ is an $\mathfrak{s - t r i a n g l e , ~ t h e n ~ t h e r e ~ i s ~ a ~ m o r p h i s m ~} g: B \rightarrow E$ which gives a morphism of $\mathfrak{s}$-triangles

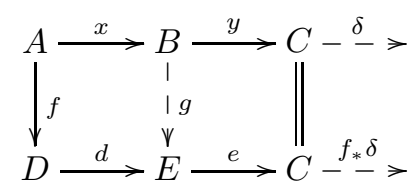

and moreover, $A \stackrel{\left(\begin{array}{c}f \\ x\end{array}\right)}{\longrightarrow} D \oplus B \stackrel{(d,-g)}{\longrightarrow} E-\stackrel{e^{*} \delta}{\rightarrow}$ is an $\mathfrak{s}$-triangle.

Lemma 2.9. (15, Corollary 3.12]) Let $\mathcal{C}$ be an extriangulated category. Then for any $\mathfrak{s}$-triangle $A \stackrel{x}{\longrightarrow} B \stackrel{y}{\longrightarrow} C-\stackrel{\delta}{\rightarrow}$, the following two sequences are exact.

$$
\begin{aligned}
& \mathcal{C}(-, A) \rightarrow \mathcal{C}(-, B) \rightarrow \mathcal{C}(-, C) \rightarrow \mathbb{E}(-, A) \rightarrow \mathbb{E}(-, B) \rightarrow \mathbb{E}(-, C), \\
& \mathcal{C}(C,-) \rightarrow \mathcal{C}(B,-) \rightarrow \mathcal{C}(A,-) \rightarrow \mathbb{E}(C,-) \rightarrow \mathbb{E}(B,-) \rightarrow \mathbb{E}(A,-) .
\end{aligned}
$$

Let $\mathcal{C}$ be an extriangulated category with enough projectives $\mathcal{P}$ and enough injectives $\mathcal{I}$. Let $X$ be any object in $\mathcal{C}$. It admits an $\mathfrak{s}$-triangle

$$
X \longrightarrow I^{0} \longrightarrow \Sigma X \stackrel{\delta^{X}}{-} \rightarrow\left(\text { resp. } \Omega X \longrightarrow{ }^{\longrightarrow} \stackrel{\delta_{X}}{\longrightarrow} \rightarrow\right)
$$

with $I^{0} \in \mathcal{I}$ (resp. $\left.P_{0} \in \mathcal{P}\right)$. We can get $\mathfrak{s}$-triangles

$$
\Sigma^{i} X \longrightarrow I^{i} \longrightarrow \Sigma^{i+1} X^{\delta^{\Sigma^{i} X}}-\rightarrow\left(\text { resp. } \Omega^{i+1} X \longrightarrow P_{i} \longrightarrow \Omega^{i} X \stackrel{\delta_{\Omega^{i}}}{-} \rightarrow\right)
$$

with $I^{i} \in \mathcal{I}$ (resp. $\left.P_{i} \in \mathcal{P}\right)$ for $i>0$ recursively.

For convenience, we denote $\mathbb{E}\left(X, \Sigma^{i} Y\right) \cong \mathbb{E}\left(\Omega^{i} X, Y\right)$ by $\mathbb{E}^{i}(X, Y)$, where the equivalence follows from [11, Lemma 5.1].

The following result extends the exact sequences appeared in Lemma 2.9.

Lemma 2.10. ([11, Proposition 5.2]) Let $\mathcal{C}$ be an extriangulated category with enough projectives $\mathcal{P}$ and enough injectives $\mathcal{I}$. Then for any $\mathfrak{s}$-triangle

$$
A \stackrel{x}{\longrightarrow} B \stackrel{y}{\longrightarrow} C-\stackrel{\delta}{-} \rightarrow
$$

the following two sequences are exact.

$$
\begin{aligned}
& \mathcal{C}(-, A) \rightarrow \mathcal{C}(-, B) \rightarrow \mathcal{C}(-, C) \rightarrow \mathbb{E}(-, A) \rightarrow \mathbb{E}(-, B) \rightarrow \mathbb{E}(-, C) \rightarrow \mathbb{E}^{2}(-, A) \\
& \quad \rightarrow \mathbb{E}^{2}(-, B) \rightarrow \mathbb{E}^{2}(-, C) \rightarrow \cdots \rightarrow \mathbb{E}^{i}(-, A) \rightarrow \mathbb{E}^{i}(-, B) \rightarrow \mathbb{E}^{i}(-, C) \rightarrow \cdots \\
& \mathcal{C}(C,-) \rightarrow \mathcal{C}(B,-) \rightarrow \mathcal{C}(A,-) \rightarrow \mathbb{E}(C,-) \rightarrow \mathbb{E}(B,-) \rightarrow \mathbb{E}(A,-) \rightarrow \mathbb{E}^{2}(C,-) \\
& \quad \rightarrow \mathbb{E}^{2}(B,-) \rightarrow \mathbb{E}^{2}(A,-) \rightarrow \cdots \rightarrow \mathbb{E}^{i}(C,-) \rightarrow \mathbb{E}^{i}(B,-) \rightarrow \mathbb{E}^{i}(A,-) \rightarrow \cdots
\end{aligned}
$$

Lemma 2.11. Let $\mathcal{C}$ be an extriangulated category with enough projectives $\mathcal{P}$ and $f: X \rightarrow Y$ be a morphism in $\mathcal{C}$.

(1) If $\pi: P \rightarrow Y$ is an $\mathfrak{s}$-deflation with $P \in \mathcal{P}$, then $(f,-\pi): X \oplus P \rightarrow Y$ is an $\mathfrak{s - d e f l a t i o n}$ and $(f,-\pi) \cong \underline{f}$ in Mor- $\mathcal{C} /[\mathcal{P}]$.

(2) If $h: X \overrightarrow{\rightarrow Z}$ is a morphism in $\mathcal{C}$ and $g: Y \rightarrow Z$ is an $\mathfrak{s}$-deflation such that $\underline{g f}=\underline{h}$ in Mor $-\mathcal{C} /[\mathcal{P}]$, then there exists an object $P \in \mathcal{P}$ and two morphisms $u: X \rightarrow P$ and $v: P \rightarrow Y$ such that $g(f-v u)=h$. 
Proof. (1) The first assertion follows from [15, Corollary 3.16] or the dual of Lemma 2.8. The second assertion is clear.

(2) Since $g f=\underline{h}$, there is an object $P \in \mathcal{P}$ and two morphisms $u: X \rightarrow P$ and $w: P \rightarrow Z$ such that $g f-h=w u$. Since $g: Y \rightarrow Z$ is an $\mathfrak{s}$-deflation, there exists a morphism $v: P \rightarrow Y$ such that $w=g v$. Therefore, $g(f-v u)=h$.

\section{Proof of Theorem 1.1}

Throughout this paper, we assume that $(\mathcal{C}, \mathbb{E}, \mathfrak{s})$ is an extriangulated category.

Let $\mathcal{M}$ be a full subcategory of $\mathcal{C}$. We denote by $\mathfrak{s}$ - $\operatorname{def}(\mathcal{M})(\operatorname{resp} \cdot \mathfrak{s}$-inf $(\mathcal{M}))$ the full subcategory of $\operatorname{Mor}(\mathcal{M})$ consisting of $\mathfrak{s}$-deflations (resp. $\mathfrak{s}$-inflations). Recall that the full subcategory of $\mathfrak{s}-\operatorname{def}(\mathcal{M})$ consisting of split epimorphisms (resp. split monomorphisms) is denoted by s-epi $(\mathcal{M})$ (resp. s-mono $(\mathcal{M}))$. We denote by sp$\operatorname{epi}(\mathcal{M})($ resp. si-mono $(\mathcal{M}))$ the full subcategory of $\mathfrak{s}$ - $\operatorname{def}(\mathcal{M})$ consisting of $(M \stackrel{1}{\rightarrow}$ $M) \oplus\left(P \rightarrow M^{\prime}\right)\left(\right.$ resp. $\left.(M \stackrel{1}{\rightarrow} M) \oplus\left(M^{\prime} \rightarrow I\right)\right)$ with $P \in \mathcal{P}($ resp. $I \in \mathcal{I})$.

Lemma 3.1. Let $\mathcal{C}$ be an extriangulated category with enough projectives $\mathcal{P}$ and $\mathcal{M}$ be a full subcategory of $\mathcal{C}$ containing $\mathcal{P}$. Assume that the following

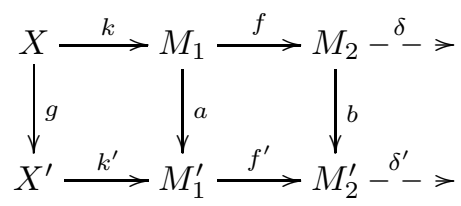

is a morphism of $\mathfrak{s}$-triangles with $M_{i}, M_{i}^{\prime} \in \mathcal{M}$. Then

(1) The following statements are equivalent.

(a) The morphism $\underline{b}$ factors through $f^{\prime}$ in $\mathcal{M} /[\mathcal{P}]$.

(b) The morphism $b$ factors through $\bar{f}^{\prime}$.

(c) The morphism $(a, b)$ factors through some object in $\mathrm{s}$-epi $(\mathcal{M})$.

(2) The following statements are equivalent.

(a) The morphism a factors through $\underline{f}$ in $\mathcal{M} /[\mathcal{P}]$.

(b) The morphism $(a, b)$ factors through some object in sp-epi $(\mathcal{M})$.

Proof. (1) Since (b) $\Leftrightarrow(c)$ follows from Lemma 2.1 and (b) $\Rightarrow(a)$ is clear, we only prove $(\mathrm{a}) \Rightarrow(\mathrm{b})$. Suppose that there is a morphism $\underline{p}: M_{2} \rightarrow M_{1}^{\prime}$ such that $\underline{f}^{\prime} \underline{p}=\underline{b}$. By Lemma 2.11, there exists an object $P \in \mathcal{P}$ and two morphisms $u: M_{2} \rightarrow \bar{P}$ and $v: P \rightarrow M_{1}^{\prime}$ such that $f^{\prime}(p-v u)=b$. Thus $b$ factors through $f^{\prime}$.

(2) (a) $\Rightarrow(\mathrm{b})$. Suppose that there is a morphism $\underline{p}: M_{2} \rightarrow M_{1}^{\prime}$ such that $\underline{p f}=\underline{a}$. Since $\mathcal{C}$ has enough projectives, there is an $\mathfrak{s}$-deflation $a_{1}: P \rightarrow M_{1}^{\prime}$ with $\bar{P} \in \overline{\mathcal{P}}$. It is easy to see that $a-p f$ factors through $a_{1}$. We assume that $a-p f=a_{1} a_{2}$ where $a_{2}: M_{1} \rightarrow P$. Since $\left(b-f^{\prime} p\right) f=f^{\prime} a-f^{\prime} p f=f^{\prime} a_{1} a_{2}$, we have the following commutative diagram.

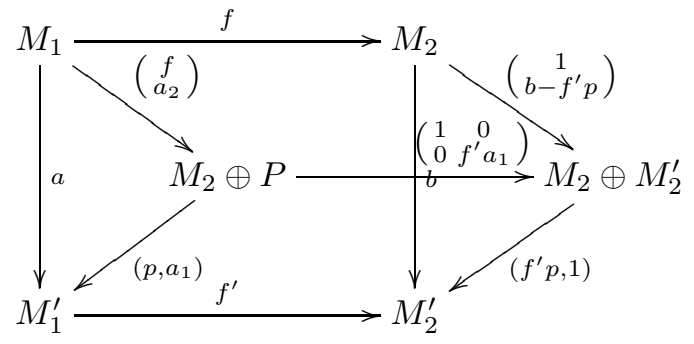


In other words, $(a, b)$ factors through $\left(M_{2} \oplus P \stackrel{\left(\begin{array}{ll}1 & 0 \\ 0 & f^{\prime} a_{1}\end{array}\right)}{\longrightarrow} M_{2} \oplus M_{2}^{\prime}\right) \in \operatorname{sp-epi}(\mathcal{M})$.

(b) $\Rightarrow(\mathrm{a})$. Assume that the morphism $(a, b)$ factors through $\left(M \oplus P \stackrel{\left(\begin{array}{ll}1 & 0 \\ 0 & \pi\end{array}\right)}{\longrightarrow}\right.$ $\left.M \oplus M^{\prime}\right) \in \operatorname{sp}-\operatorname{epi}(\mathcal{M})$. Suppose that the following diagram

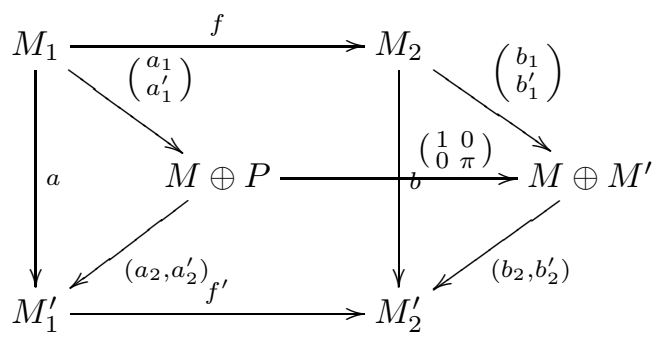

is commutative. Let $p=a_{2} b_{1}: M_{2} \rightarrow M_{1}^{\prime}$, then $p f=a_{2} b_{1} f=a_{2} a_{1}$, thus $\underline{a}=$ $\underline{a_{2} a_{1}}=\underline{p f}$.

Theorem 3.2. Let $\mathcal{C}$ be an extriangulated category and $\mathcal{M}$ be a full subcategory of $\mathcal{C}$.

(1) If $\mathcal{C}$ has enough projectives $\mathcal{P}$ and $\mathcal{M}$ contains $\mathcal{P}$, then $\mathfrak{s}-\operatorname{def}(\mathcal{M}) /[\operatorname{s-epi}(\mathcal{M})] \cong$ $\bmod -\mathcal{M} /[\mathcal{P}]$ and $\mathfrak{s}-\operatorname{def}(\mathcal{M}) /[\operatorname{sp}-\operatorname{epi}(\mathcal{M})] \cong\left(\bmod -(\mathcal{M} /[\mathcal{P}])^{\mathrm{op}}\right)^{\mathrm{op}}$.

(2) If $\mathcal{C}$ has enough injectives $\mathcal{I}$ and $\mathcal{M}$ contains $\mathcal{I}$, then $\mathfrak{s}-\inf (\mathcal{M}) /[\operatorname{s-mono}(\mathcal{M})] \cong$ $\left(\bmod -(\mathcal{M} /[\mathcal{I}])^{\text {op }}\right)^{\text {op }}$ and $\mathfrak{s}-\inf (\mathcal{M}) /[\operatorname{si}-\operatorname{mono}(\mathcal{M})] \cong \bmod -\mathcal{M} /[\mathcal{I}]$.

Proof. Since (2) is dual to (1), we only prove (1).

Define a functor

$$
F: \mathfrak{s}-\operatorname{def}(\mathcal{M}) \rightarrow \operatorname{Mor}(\mathcal{M} /[\mathcal{P}]), \quad\left(M_{1} \stackrel{f}{\rightarrow} M_{2}\right) \mapsto\left(M_{1} \stackrel{f}{\rightarrow} M_{2}\right) .
$$

For any object $f: M_{1} \rightarrow M_{2}$ in $\operatorname{Mor}(\mathcal{M} /[\mathcal{P}])$, by Lemma 2.11 there is an object $P \in \mathcal{P}$ and an $\mathfrak{s}$-deflation $(f,-\pi): M_{1} \oplus P \rightarrow M_{2}$ such that $(f,-\pi) \cong \underline{f}$. Therefore, $F(f,-\pi) \cong f$ and $F$ is dense.

Assume that $f: M_{1} \rightarrow M_{2}$ and $f^{\prime}: M_{1}^{\prime} \rightarrow M_{2}^{\prime}$ are objects in $\mathfrak{s}-\operatorname{def}(\mathcal{M})$ and $(\underline{a}, \underline{b})$ is a morphism in $\operatorname{Mor}(\mathcal{M} /[\mathcal{P}])$ from $f$ to $f^{\prime}$. Then $\underline{b} f=f^{\prime} \underline{a}$. By Lemma 2.11 there exists an object $Q \in \mathcal{P}$ and two morphisms $u: M_{1} \rightarrow Q$ and $v: Q \rightarrow M_{1}^{\prime}$ such that $f^{\prime}(a-v u)=b f$. Thus, $F(a-v u, b)=(\underline{a}, \underline{b})$ and the functor $F$ is full.

The functor $F$ induces a full and dense functor $\widetilde{F}: \mathfrak{s}$-def $(\mathcal{M}) \rightarrow \operatorname{Mor}(\mathcal{M} /[\mathcal{P}]) / \mathcal{R}$. By Lemma 3.1 $(1)$, we have $\mathfrak{s}-\operatorname{def}(\mathcal{M}) /[\operatorname{s-epi}(\mathcal{C})] \cong \operatorname{Mor}(\mathcal{M} /[\mathcal{P}]) / \mathcal{R}$. It follows that $\mathfrak{s}-\operatorname{def}(\mathcal{M}) /[\operatorname{s-epi}(\mathcal{M})] \cong \bmod -\mathcal{M} /[\mathcal{P}]$ by Lemma $2.1(1)$.

The functor $F$ induces a full and dense functor $\widehat{F}: \mathfrak{s}-\operatorname{def}(\mathcal{M}) \rightarrow \operatorname{Mor}(\mathcal{M} /[\mathcal{P}]) / \mathcal{R}^{\prime}$. By Lemma 3.1 $(2)$, we have $\mathfrak{s}-\operatorname{def}(\mathcal{M}) /[\operatorname{sp}-\operatorname{epi}(\mathcal{C})] \cong \operatorname{Mor}(\mathcal{M} /[\mathcal{P}]) / \mathcal{R}^{\prime}$. It follows that $\mathfrak{s}-\operatorname{def}(\mathcal{M}) /[\operatorname{sp}-\operatorname{epi}(\mathcal{M})] \cong\left(\bmod -(\mathcal{M} /[\mathcal{P}])^{\text {op }}\right)^{\text {op }}$ by Lemma $2.1(2)$.

\section{Application to CATEgory of $\mathfrak{s}$-TRIAngles}

In this section, we will investigate the first application of Theorem 3.2 in the case when $\mathcal{M}=\mathcal{C}$.

We denote by $\mathfrak{s}$-tri $(\mathcal{C})$ the category of $\mathfrak{s}$-triangles in $\mathcal{C}$, where the objects are the $\mathfrak{s}$-triangles $X_{\bullet}=\left(X_{1} \stackrel{f_{1}}{\longrightarrow} X_{2} \stackrel{f_{2}}{\longrightarrow} X_{3}-\stackrel{\delta}{-}>\right)$ and the morphisms from $X_{\bullet}$ to 
$Y_{\bullet}$ are the triples $\varphi_{\bullet}=\left(\varphi_{1}, \varphi_{2}, \varphi_{3}\right)$ such that the following diagram is commutative

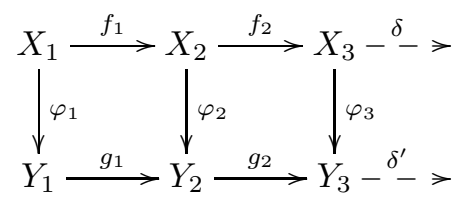

and $\varphi_{1 *} \delta=\varphi_{3}^{*} \delta^{\prime}$. Let $X_{\bullet}$ and $Y_{\bullet}$ be two $\mathfrak{s - t r i a n g l e s , ~ w e ~ d e n o t e ~ b y ~} \mathcal{R}_{2}\left(X_{\bullet}, Y_{\bullet}\right)$ (resp. $\left.\mathcal{R}_{1}^{\prime}\left(X_{\bullet}, Y_{\bullet}\right)\right)$ the class of morphisms $\varphi_{\bullet}: X_{\bullet} \rightarrow Y_{\bullet}$ such that $\varphi_{3}$ factors through $g_{2}$ (resp. $\varphi_{1}$ factors through $f_{1}$ ). It is easy to see that $\mathcal{R}_{2}$ and $\mathcal{R}_{1}^{\prime}$ are ideals of $\mathfrak{s}$-tri $(\mathcal{C})$.

Theorem 4.1. Let $\mathcal{C}$ be an extriangulated category.

(1) If $\mathcal{C}$ has enough projectives $\mathcal{P}$, then $\mathfrak{s}$ - $\operatorname{tri}(\mathcal{C}) / \mathcal{R}_{2} \cong \bmod -\mathcal{C} /[\mathcal{P}]$.

(2) If $\mathcal{C}$ has enough injectives $\mathcal{I}$, then $\mathfrak{s}$ - $\operatorname{tri}(\mathcal{C}) / \mathcal{R}_{2} \cong\left(\bmod -(\mathcal{C} /[\mathcal{I}])^{\mathrm{op}}\right)^{\mathrm{op}}$.

Proof. (1) We have $\mathfrak{s}$-tri $(\mathcal{C}) / \mathcal{R}_{2} \cong \mathfrak{s}-\operatorname{def}(\mathcal{C}) /[\operatorname{s-epi}(\mathcal{C})]$ by Lemma 3.1] Thus $\mathfrak{s}$-tri $(\mathcal{C}) / \mathcal{R}_{2} \cong$ mod- $\mathcal{C} /[\mathcal{P}]$ follows from Theorem $3.2(1)$.

(2) We note that $\mathcal{R}_{2}=\mathcal{R}_{1}^{\prime}$ by Lemma 2.7. Thus $\mathfrak{s}$ - $\operatorname{tri}(\mathcal{C}) / \mathcal{R}_{2}=\mathfrak{s}$-tri $(\mathcal{C}) / \mathcal{R}_{1}^{\prime} \cong$ $\mathfrak{s}-\inf (\mathcal{C}) /[\mathrm{s}-\operatorname{mono}(\mathcal{C})] \cong\left(\bmod -(\mathcal{C} /[\mathcal{I}])^{\text {op }}\right)^{\text {op }}$, where the last equivalence follows from Theorem $3.2(2)$.

Lemma 4.2. Let $\mathcal{C}$ be an extriangulated category. Assume that the following

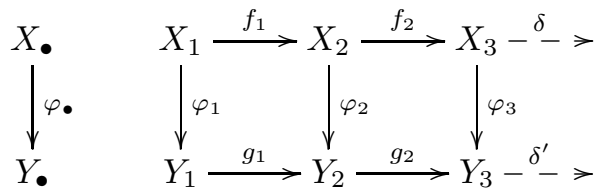

is a morphism of $\mathfrak{s}$-triangles. Then

(1) The following statements are equivalent.

(a) $\varphi_{\bullet}=0$ in $\mathfrak{s}-\operatorname{tri}(\mathcal{C}) / \mathcal{R}_{2}$.

(b) $\overline{\varphi_{1}}$ factors through $f_{1}$.

(c) $\varphi_{3}$ factors through $g_{2}$.

(2) The following statements are equivalent.

(a) $\varphi$ • is a monomorphism in $\mathfrak{s}$-tri $(\mathcal{C}) / \mathcal{R}_{2}$.

(b) $\left(\begin{array}{l}f_{1} \\ \varphi_{1}\end{array}\right): X_{1} \rightarrow X_{2} \oplus Y_{1}$ is a section.

Proof. (1) It follows from Lemma 2.7.

(2) The proof is similar to [10, Lemma 4.7].

If $\mathcal{C}$ has enough projectives $\mathcal{P}$, then $\mathfrak{s}$ - $\operatorname{tri}(\mathcal{C}) / \mathcal{R}_{2} \cong \bmod -\mathcal{C} /[\mathcal{P}]$ is abelian by Theorem 4.1. The following result implies that $\mathfrak{s}$ - $\operatorname{tri}(\mathcal{C}) / \mathcal{R}_{2}$ is always abelian for general case.

Proposition 4.3. Let $\mathcal{C}$ be an extriangulated category. Then $\mathfrak{s}$-tri $(\mathcal{C}) / \mathcal{R}_{2}$ is an abelian category.

Proof. The proof is an adaption of [10, Theorem 4.8]. Assume that the following

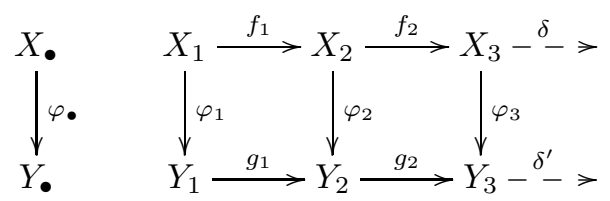


is a morphism of $\mathfrak{s}$-triangles. Thus $\varphi_{1 *} \delta=\varphi_{3}^{*} \delta^{\prime}$ by definition. By Lemma 2.8 and its dual, we have the following morphisms of $\mathfrak{s}$-triangles.

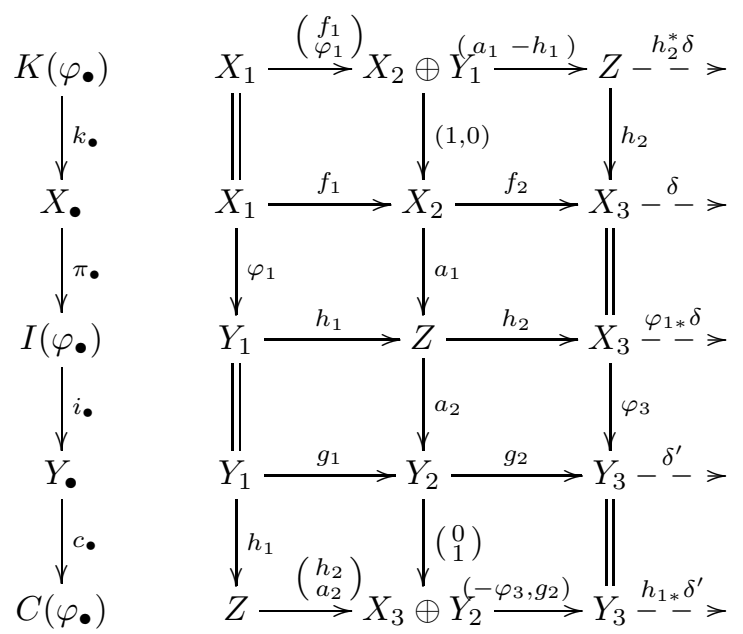

Moreover, we have $\varphi_{\bullet}=i_{\bullet} \pi_{\bullet}$ in $\mathfrak{s}$-tri $(\mathcal{C}) / \mathcal{R}_{2}$. It is routine to check that $k_{\bullet}$ : $K\left(\varphi_{\bullet}\right) \rightarrow X_{\bullet}$ is a kernel of $\underline{\varphi_{\bullet}}, c_{\bullet}: Y_{\bullet} \rightarrow C\left(\varphi_{\bullet}\right)$ is a cokernel of $\underline{\varphi_{\bullet}}$ and

$$
\operatorname{Coker}\left(\operatorname{Ker}\left(\underline{\varphi_{\bullet}}\right)\right) \cong I\left(\varphi_{\bullet}\right) \cong \operatorname{Ker}\left(\operatorname{Coker}\left(\underline{\varphi_{\bullet}}\right)\right) .
$$

Proposition 4.4. Let $\mathcal{C}$ be an extriangulated category. Then an $\mathfrak{s - t r i a n g l e ~} P_{X}=$ $\left(\Omega X \stackrel{f_{1}}{\longrightarrow} P \stackrel{f_{2}}{\longrightarrow} X-\stackrel{\rho}{\longrightarrow}\right)$ ) with $P \in \mathcal{P}$ is a projective object in $\mathfrak{s}$-tri $(\mathcal{C}) / \mathcal{R}_{2}$. Moreover, if $\mathcal{C}$ has enough projectives, then each projective object in $\mathfrak{s}$-tri $(\mathcal{C}) / \mathcal{R}_{2}$ is of the form $P_{X}$.

Proof. The proof is an adaption of [10, Proposition 4.11]. We omit it.

Definition 4.5. (6, [17]) An $\mathfrak{s - t r i a n g l e ~} X_{1} \stackrel{f_{1}}{\longrightarrow} X_{2} \stackrel{f_{2}}{\longrightarrow} X_{3}-{ }^{\delta} \rightarrow$ is called Auslander-Reiten $\mathfrak{s}$-triangle if the following holds:

(1) $\delta \in \mathbb{E}(C, A)$ is non-split.

(2) If $g: X_{1} \rightarrow Y$ is not a section, then $g$ factors through $f_{1}$.

(3) If $h: Z \rightarrow X_{3}$ is not a retraction, then $h$ factors through $f_{2}$.

Proposition 4.6. Let $\mathcal{C}$ be a Krull-Smidt extriangulated category. Assume that $X_{\bullet}: X_{1} \stackrel{f_{1}}{\longrightarrow} X_{2} \stackrel{f_{2}}{\longrightarrow} X_{3}-\stackrel{\delta}{-} \quad$ is a non-split $\mathfrak{s}$-triangle such that $X_{1}$ and $X_{2}$ are indecomposable. Then $X_{\bullet}$ is a simple object in $\mathfrak{s - t r i}(\mathcal{C}) / \mathcal{R}_{2}$ if and only if $X_{\bullet}$ is an Auslander-Reiten $\mathfrak{s}$-triangle in $\mathcal{C}$.

Proof. The proof is an adaption of [10, Theorem 4.20 (a)].

From now on to the end of this section we assume that $\mathcal{C}$ is an extriangulated category with enough projectives $\mathcal{P}$ and enough injectives $\mathcal{I}$.

Given an $\mathfrak{s}$-triangle $\delta=\left(X_{1} \stackrel{f_{1}}{\longrightarrow} X_{2} \stackrel{f_{2}}{\longrightarrow} X_{3}-\stackrel{\rho}{-}>\right)$, we define the contravariant defect $\delta^{*}$ and the covariant defect $\delta_{*}$ by the following exact sequence of functors

$$
\mathcal{C}\left(-, X_{1}\right) \stackrel{\mathcal{C}\left(-, f_{1}\right)}{\longrightarrow} \mathcal{C}\left(-, X_{2}\right) \stackrel{\mathcal{C}\left(-, f_{2}\right)}{\longrightarrow} \mathcal{C}\left(-, X_{3}\right) \rightarrow \delta^{*} \rightarrow 0
$$




$$
\mathcal{C}\left(X_{3},-\right) \stackrel{\mathcal{C}\left(f_{2},-\right)}{\longrightarrow} \mathcal{C}\left(X_{2},-\right) \stackrel{\mathcal{C}\left(f_{1},-\right)}{\longrightarrow} \mathcal{C}\left(X_{1},-\right) \rightarrow \delta_{*} \rightarrow 0
$$

Example 4.7. (1) Let $\delta=P_{X}=\left(\Omega X \stackrel{f_{1}}{\longrightarrow} P \stackrel{f_{2}}{\longrightarrow} X-\stackrel{\rho}{\longrightarrow}\right)$ ) with $P \in \mathcal{P}$. Then $\delta^{*}=\mathcal{C} /[\mathcal{P}](-, X)$ and $\delta_{*}=\mathbb{E}(X,-)$.

(2) Let $\delta=I_{X}=\left(X \stackrel{f_{1}}{\longrightarrow} I \stackrel{f_{2}}{\longrightarrow} \Sigma X \stackrel{\rho}{-} \rightarrow^{\longrightarrow}\right)$ with $I \in \mathcal{I}$. Then $\delta^{*}=\mathbb{E}(-, X)$ and $\delta_{*}=\mathcal{C} /[\mathcal{I}](X,-)$.

The following result gives an explanation of [6, Theorem 4.1].

Theorem 4.8. Let $\mathcal{C}$ be an extriangulated category with enough projectives $\mathcal{P}$ and enough injectives $\mathcal{I}$.

(1) We have the following equivalences

$$
\mathfrak{s}-\operatorname{tri}(\mathcal{C}) / \mathcal{R}_{2} \cong \bmod -\mathcal{C} /[\mathcal{P}] \cong\left(\bmod -(\mathcal{C} /[\mathcal{I}])^{\text {op }}\right)^{\text {op }}
$$

Moreover, the equivalence $F: \mathfrak{s}$-tri $(\mathcal{C}) / \mathcal{R}_{2} \cong \bmod -\mathcal{C} /[\mathcal{P}]$ is given by $\delta \mapsto \delta^{*}$ and the equivalence $G: \mathfrak{s}$ - $\operatorname{tri}(\mathcal{C}) / \mathcal{R}_{2} \cong\left(\bmod -(\mathcal{C} /[\mathcal{I}])^{\text {op }}\right)^{\text {op }}$ is given by $\delta \mapsto \delta_{*}$.

(2) The abelian category mod-C $/[\mathcal{P}]$ has enough projectives and enough injectives. Moreover, each projective object is of the form $\mathcal{C} /[\mathcal{P}](-, X)$, and each injective object is of the form $\mathbb{E}(-, X)$.

(3) The abelian category mod- $(\mathcal{C} /[\mathcal{I}])^{\text {op }}$ has enough projectives and enough injectives. Moreover, each projective object is of the form $\mathcal{C} /[\mathcal{I}](X,-)$, and each injective object is of the form $\mathbb{E}(X,-)$.

Proof. (1) The first assertion follows from Theorem 4.1. Assume that

$$
\delta=\left(X_{1} \stackrel{f_{1}}{\longrightarrow} X_{2} \stackrel{f_{2}}{\longrightarrow} X_{3}-\stackrel{\rho}{-} \rightarrow\right)
$$

is an $\mathfrak{s}$-triangle. Recall that $F(\delta)=\operatorname{Coker}\left(\mathcal{C} /[\mathcal{P}]\left(-, f_{2}\right)\right)$ and $\delta^{*}=\operatorname{Coker}\left(\mathcal{C}\left(-, f_{2}\right)\right)$. Since $\delta^{*}(\mathcal{P})=0$, we can view $\delta^{*}$ as a finitely presented $\mathcal{C} /[\mathcal{P}]$-module. Thus $F(\delta)=$ $\delta^{*}$. Similarly, we have $G(\delta)=\delta_{*}$.

(2) and (3) follows from (1), Proposition 4.4 and Example 4.7.

Corollary 4.9. Let $\mathcal{C}$ be an extriangulated category with enough projectives $\mathcal{P}$ and enough injectives $\mathcal{I}$. Then there is a duality

$$
\Phi: \bmod -\mathcal{C} /[\mathcal{P}] \rightarrow \bmod -(\mathcal{C} /[\mathcal{I}])^{\text {op }}, \quad \delta^{*} \mapsto \delta_{*} .
$$

Moreover, by restrictions, we obtain the following two dualities

$$
\begin{aligned}
& \Phi: \operatorname{proj}-\mathcal{C} /[\mathcal{P}] \rightarrow \operatorname{inj}-(\mathcal{C} /[\mathcal{I}])^{\text {op }}, \quad \mathcal{C} /[\mathcal{P}](-, X) \mapsto \mathbb{E}(X,-) . \\
& \Phi: \operatorname{inj}-\mathcal{C} /[\mathcal{P}] \rightarrow \operatorname{proj}-(\mathcal{C} /[\mathcal{I}])^{\text {op }}, \quad \mathbb{E}(-, X) \mapsto \mathcal{C} /[\mathcal{I}](X,-) .
\end{aligned}
$$

Proof. It is a direct consequence of Theorem 4.8 .

Corollary 4.10. (6, Proposition 4.9]) Let $\mathcal{C}$ be an extriangulated category with enough projectives $\mathcal{P}$ and enough injectives $\mathcal{I}$.

(1) There is an isomorphism between $\mathcal{C} /[\mathcal{P}](Y, X)$ and the group of natural transformations from $\mathbb{E}(X,-)$ to $\mathbb{E}(Y,-)$.

(2) There is an isomorphism between $\mathcal{C} /[\mathcal{I}](X, Y)$ and the group of natural transformations from $\mathbb{E}(-, X)$ to $\mathbb{E}(-, Y)$.

Now we have the following Auslander-Reiten duality and defect formula for extriangulated categories. 
Proposition 4.11. Let $\mathcal{C}$ be an Ext-finite extriangulated category with enough projectives $\mathcal{P}$ and enough injectives $\mathcal{I}$. Assume that either $\mathcal{C} /[\mathcal{P}]$ or $\mathcal{C} /[\mathcal{I}]$ is a dualizing $k$-variety. Then there is an equivalence $\tau: \mathcal{C} /[\mathcal{P}] \cong \mathcal{C} /[\mathcal{I}]$ satisfying the following properties:

(1) $D \mathbb{E}(-, X) \cong \mathcal{C} /[\mathcal{P}]\left(\tau^{-1} X,-\right), D \mathbb{E}(X,-) \cong \mathcal{C} /[\mathcal{I}](-, \tau X)$.

(2) $D \delta_{*}=\delta^{*} \tau^{-1}, D \delta^{*}=\delta_{*} \tau$ for each $\mathfrak{s - t r i a n g l e ~} \delta$.

Proof. Without loss of generality, we assume that $\mathcal{C} /[\mathcal{I}]$ is a dualizing $k$-variety. The composition of $\Phi: \bmod -\mathcal{C} /[\mathcal{P}] \rightarrow \bmod -(\mathcal{C} /[\mathcal{I}])^{\text {op }}$ and $D: \bmod -(\mathcal{C} /[\mathcal{I}])^{\text {op }} \rightarrow$ $\bmod -\mathcal{C} /[\mathcal{I}]$ defines an equivalence

$$
\Theta: \bmod -\mathcal{C} /[\mathcal{P}] \stackrel{\Phi}{\rightarrow} \bmod -(\mathcal{C} /[\mathcal{I}])^{\text {op }} \stackrel{D}{\rightarrow} \bmod -\mathcal{C} /[\mathcal{I}] .
$$

It follows that $\Theta(\mathcal{C} /[\mathcal{P}](-, X))=D \mathbb{E}(X,-) \cong \mathcal{C} /[\mathcal{I}](-, Y)$ for some $Y \in \mathcal{C}$. Therefore, there is an equivalence $\tau: \mathcal{C} /[\mathcal{P}] \cong \mathcal{C} /[\mathcal{I}]$ mapping $X$ to $Y$. The equivalence $\tau$ induces an equivalence $\tau_{*}^{-1}: \bmod -\mathcal{C} /[\mathcal{P}] \cong \bmod -\mathcal{C} /[\mathcal{I}], F \mapsto F \tau^{-1}$, such that $D \Phi=\tau_{*}^{-1}$. Assume that $\delta$ is an $\mathfrak{s}$-triangle, then $D \Phi\left(\delta^{*}\right)=D \delta_{*}$. On the other hand, $\tau_{*}^{-1}\left(\delta^{*}\right)=\delta^{*} \tau^{-1}$. Hence, we have $D \delta_{*}=\delta^{*} \tau^{-1}$. It follows that $D \delta^{*}=\delta_{*} \tau$. If $\delta=I_{X}$, then by Example 4.7 we have $\delta^{*}=\mathbb{E}(-, X)$ and $\delta_{*}=\mathcal{C} / \mathcal{I}(X,-)$. Therefore, we have $D \mathbb{E}(-, X) \cong \mathcal{C} /[\mathcal{I}](X, \tau-) \cong \mathcal{C} /[\mathcal{P}]\left(\tau^{-1} X,-\right)$ since $D \delta^{*}=\delta_{*} \tau$.

\section{Application to Rigid SUbCategories}

In this section, we will investigate the second application of Theorem 3.2 in the case when $\mathcal{M}$ is a rigid subcategory of $\mathcal{C}$, that is, $\mathbb{E}\left(M, M^{\prime}\right)=0$ for any objects $M, M^{\prime} \in \mathcal{M}$.

Let $\mathcal{C}^{\prime}$ and $\mathcal{C}^{\prime \prime}$ be two full subcategories of $\mathcal{C}$. We denote by Cocone $\left(\mathcal{C}^{\prime}, \mathcal{C}^{\prime \prime}\right)$ the full subcategory of $\mathcal{C}$ of objects $X$ admitting an $\mathfrak{s}$-triangle $X \longrightarrow C^{\prime} \longrightarrow C^{\prime \prime}-->$ with $C^{\prime} \in \mathcal{C}^{\prime}$ and $C^{\prime \prime} \in \mathcal{C}^{\prime \prime}$. We denote by $\operatorname{Cone}\left(\mathcal{C}^{\prime}, \mathcal{C}^{\prime \prime}\right)$ the full subcategory of objects $X$ admitting an $\mathfrak{s}$-triangle $C^{\prime} \longrightarrow C^{\prime \prime} \longrightarrow X--\longrightarrow$ with $C^{\prime} \in \mathcal{C}^{\prime}$ and $C^{\prime \prime} \in \mathcal{C}^{\prime \prime}$.

For convenience we let $\mathcal{M}_{L}=\operatorname{Cocone}(\mathcal{M}, \mathcal{M})$ and $\mathcal{M}_{R}=\operatorname{Cone}(\mathcal{M}, \mathcal{M})$. If $\mathcal{C}$ has enough projectives $\mathcal{P}$, then we let $\Omega \mathcal{M}=\operatorname{Cocone}(\mathcal{P}, \mathcal{M})$. If $\mathcal{C}$ has enough injectives $\mathcal{I}$, then we let $\Sigma \mathcal{M}=\operatorname{Cone}(\mathcal{M}, \mathcal{I})$.

Lemma 5.1. Let $\mathcal{C}$ be an extriangulated category and $\mathcal{M}$ be a rigid subcategory of C. If $X \stackrel{k}{\longrightarrow} M_{1} \stackrel{f}{\longrightarrow} M_{2}-{ }_{-} \rightarrow$ is an $\mathfrak{s}$-triangle with $M_{i} \in \mathcal{M}$, then $k$ is a left $\mathcal{M}$-approximation of $X$.

Proof. For any $M \in \mathcal{M}$, by Lemma 2.9 we have the following exact sequence

$$
\mathcal{C}\left(M_{2}, M\right) \rightarrow \mathcal{C}\left(M_{1}, M\right) \rightarrow \mathcal{C}(X, M) \rightarrow \mathbb{E}\left(M_{2}, M\right)=0 .
$$

Hence, $k$ is a left $\mathcal{M}$-approximation of $X$.

Lemma 5.2. Let $\mathcal{C}$ be an extriangulated category with enough projectives $\mathcal{P}$ and $\mathcal{M}$ be a rigid subcategory of $\mathcal{C}$ containing $\mathcal{P}$. Assume that the following diagram

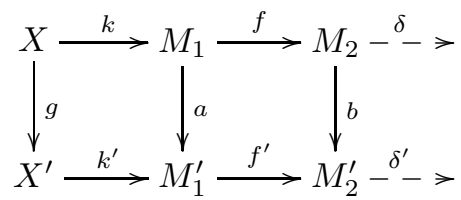


is a morphism of $\mathfrak{s}$-triangles with $M_{i}, M_{i}^{\prime} \in \mathcal{M}$. Then

(1) The following statements are equivalent.

(a) The morphism $b$ factors through $f^{\prime}$.

(b) The morphism $(a, b)$ factors through some object in s-epi $(\mathcal{M})$.

(c) The morphism $g$ factors through some object in $\mathcal{M}$.

(2) The following statements are equivalent.

(a) The morphism a factors through $f$ in $\mathcal{M} /[\mathcal{P}]$.

(b) The morphism $(a, b)$ factors through some object in sp-epi $(\mathcal{M})$.

(c) The morphism $g$ factors through some object in $\Omega \mathcal{M}$.

Proof. (1) We note that (a) $\Leftrightarrow$ (b) follows from Lemma 3.1(1).

$(\mathrm{a}) \Rightarrow(\mathrm{c})$. Assume that $b$ factors through $f^{\prime}$. It follows that $g$ factors through $k$ by Lemma 2.7 which implies that $g$ factors through $M_{1} \in \mathcal{M}$.

(c) $\Rightarrow\left(\right.$ a). Suppose that $g$ has a factorization $X \stackrel{g_{1}}{\longrightarrow} M \stackrel{g_{2}}{\longrightarrow} X^{\prime}$ with $M \in \mathcal{M}$. By Lemma 5.1 we have $g_{1}$ factors through $k$. Thus $g$ factors through $k$. It follows that $b$ factors through $f^{\prime}$ by Lemma 2.7

(2) We note that $(\mathrm{a}) \Leftrightarrow(\mathrm{b})$ follows from Lemma 3.1 (2).

(a) $\Rightarrow(\mathrm{c})$. Suppose that there is a morphism $\underline{p}: M_{2} \rightarrow M_{1}^{\prime}$ such that $\underline{p f}=\underline{a}$. Since $\mathcal{C}$ has enough projectives, there is an $\mathfrak{s}$-triangle $\Omega M_{1}^{\prime} \stackrel{a_{1}^{\prime}}{\longrightarrow} P \stackrel{a_{1}}{\longrightarrow} M_{1}^{\prime} \stackrel{\delta}{\prime \prime}_{-}^{\rightarrow}$ with $P \in \mathcal{P}$. It is easy to see that $p f-a$ factors through $a_{1}$. Assume that $p f-a=a_{1} a_{2}$ where $a_{2}: M_{1} \rightarrow P$. By $(\mathrm{ET} 4)^{\mathrm{op}}$, we have the following diagram

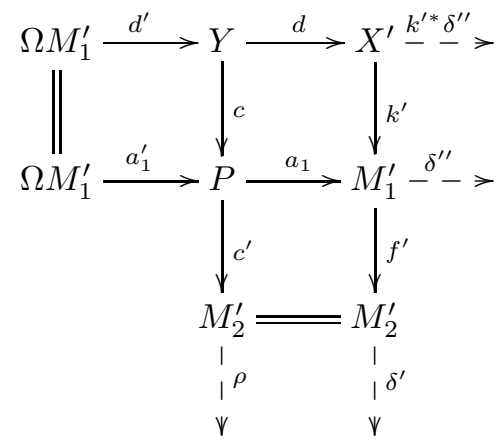

where the first row and the second column are $\mathfrak{s - t r i a n g l e s . ~ I t ~ f o l l o w s ~ t h a t ~} Y \in \Omega \mathcal{M}$. Since the upper-right square of the above diagram obtained by (ET4) ${ }^{\text {op }}$ is a weak pullback and $\left(k^{\prime}, a_{1}\right)\left(\begin{array}{c}g \\ a_{2} k\end{array}\right)=a k+a_{1} a_{2} k=p f k=0$, there exists a morphism $h: X \rightarrow Y$ such that $d h=g$ and $c h=a_{2} k$. Therefore, $g$ factors through $Y \in \Omega \mathcal{M}$.

(c) $\Rightarrow$ (a). Suppose that $g$ has a factorization $X \stackrel{g_{1}}{\longrightarrow} \Omega M \stackrel{g_{2}}{\longrightarrow} X^{\prime}$ with $\Omega M \in \Omega \mathcal{M}$. Then by Lemma 5.1 and (ET3) we complete the following morphism of $\mathfrak{s}$-triangles

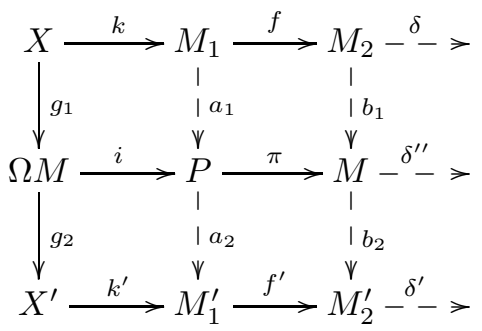


where $P \in \mathcal{P}$. Since $\left(a-a_{2} a_{1}\right) k=k^{\prime}\left(g-g_{2} g_{1}\right)=0$, there exists a morphism $p: M_{2} \rightarrow M_{1}^{\prime}$ such that $a-a_{2} a_{1}=p f$. Therefore, $\underline{a}=\underline{p f}$.

Lemma 5.3. Let $\mathcal{C}$ be an extriangulated category with enough projectives $\mathcal{P}$ and $\mathcal{M}$ be a rigid subcategory of $\mathcal{C}$ containing $\mathcal{P}$. Then

(1) $\mathfrak{s}-\operatorname{def}(\mathcal{M}) /[\operatorname{s-epi}(\mathcal{M})] \cong \mathcal{M}_{L} /[\mathcal{M}]$

(2) $\mathfrak{s - d e f}(\mathcal{M}) /[\operatorname{sp}-\operatorname{epi}(\mathcal{M})] \cong \mathcal{M}_{L} /[\Omega \mathcal{M}]$.

Proof. (1) For any object $f: M_{1} \rightarrow M_{2}$ in $\mathfrak{s}$-def $(\mathcal{M})$, there exists an $\mathfrak{s}$-triangle $X \stackrel{k}{\longrightarrow} M_{1} \stackrel{f}{\longrightarrow} M_{2}-_{-}^{\delta}>$ where $X$ is unique under isomorphism. By (ET3) ${ }^{\mathrm{op}}$, the following commutative diagram

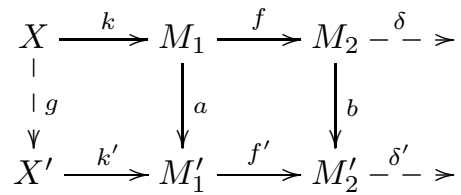

whose rows are $\mathfrak{s}$-triangles can be completed to a morphism of $\mathfrak{s}$-triangles. The morphism $g: X \rightarrow X^{\prime}$ is not unique in general. Assume that $g^{\prime}: X \rightarrow X^{\prime}$ is another morphism such that $\left(g^{\prime}, a, b\right)$ is a morphism of $\mathfrak{s}$-triangles. Then $\left(g-g^{\prime}, 0,0\right)$ is also a morphism of $\mathfrak{s}$-triangles. Lemma 2.7 implies that $g-g^{\prime}$ factors through $k$, that is, $g-g^{\prime}$ factors through $M_{1} \in \mathcal{M}$. It follows that $\underline{g}=\underline{g}^{\prime}$ in $\mathcal{M}_{L} /[\mathcal{M}]$.

Hence the assignment $\left(M_{1} \stackrel{f}{\rightarrow} M_{2}\right) \mapsto X,(a, b) \mapsto \underline{g}$ defines a well-defined functor $F: \mathfrak{s}-\operatorname{def}(\mathcal{M}) \rightarrow \mathcal{M}_{L} /[\mathcal{M}]$. It is clear that $F$ is dense. The functor $F$ is full by Lemma 5.1 and (ET3). Lemma 5.2(1) implies that $F$ induces an equivalence $\mathfrak{s}-\operatorname{def}(\mathcal{M}) / \mathcal{R} \cong \mathcal{M}_{L} /[\mathcal{M}]$, that is, $\mathfrak{s}-\operatorname{def}(\mathcal{M}) /[\operatorname{sepi}(\mathcal{M})] \cong \mathcal{M}_{L} /[\mathcal{M}]$.

(2) For any $X \in \mathcal{M}_{L}$, we fix an $\mathfrak{s - t r i a n g l e ~} X \stackrel{k}{\longrightarrow} M_{1} \stackrel{f}{\longrightarrow} M_{2}-{ }_{-}->$with $M_{1}, M_{2} \in \mathcal{M}$. Assume that $g: X \rightarrow X^{\prime}$ is a morphism in $\mathcal{M}_{L}$. Then by Lemma 5.1 and (ET3) there exist two morphisms $a: M_{1} \rightarrow M_{1}^{\prime}$ and $b: M_{2} \rightarrow M_{2}^{\prime}$ such that the following is a morphism of $\mathfrak{s}$-triangles

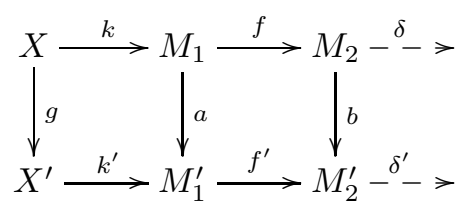

where $M_{1}^{\prime}, M_{2}^{\prime} \in \mathcal{M}$. Suppose that the morphisms $a^{\prime}: M_{1} \rightarrow M_{1}^{\prime}$ and $b^{\prime}: M_{2} \rightarrow M_{2}^{\prime}$ satisfying $\left(g, a^{\prime}, b^{\prime}\right)$ is also a morphism of $\mathfrak{s}$-triangles. Then $\left(0, a-a^{\prime}, b-b^{\prime}\right)$ is a morphism of $\mathfrak{s - t r i a n g l e s . ~ I t ~ f o l l o w s ~ t h a t ~}\left(a-a^{\prime}, b-b^{\prime}\right)$ factors through some object in sp-epi $(\mathcal{M})$ by Lemma $\left[5.2(2)\right.$. We have $(a, b)=\left(a^{\prime}, b^{\prime}\right)$ in $\mathfrak{s}$-def $(\mathcal{M}) /[\operatorname{sp}-\operatorname{epi}(\mathcal{M})]$.

Therefore, the assignment $X \mapsto\left(M_{1} \stackrel{f}{\rightarrow} M_{2}\right), g \mapsto(a, b)$ defines a well-defined functor $G: \mathcal{M}_{L} \rightarrow \mathfrak{s}$-def $(\mathcal{M}) /[\operatorname{sp}-\operatorname{epi}(\mathcal{M})]$.

It is easy to see that $G$ is full and dense. Lemma 5.2(2) implies that $G$ induces an equivalence $\mathcal{M}_{L} /[\Omega \mathcal{M}] \cong \mathfrak{s}-\operatorname{def}(\mathcal{M}) /[\operatorname{sp}-\operatorname{epi}(\mathcal{M})]$.

Theorem 5.4. Let $\mathcal{C}$ be an extriangulated category and $\mathcal{M}$ be a rigid subcategory of $\mathcal{C}$.

(1) If $\mathcal{C}$ has enough projectives $\mathcal{P}$ and $\mathcal{M}$ contains $\mathcal{P}$, then $\mathcal{M}_{L} /[\mathcal{M}] \cong \bmod -(\mathcal{M} /[\mathcal{P}])$ and $\mathcal{M}_{L} /[\Omega \mathcal{M}] \cong\left(\bmod -(\mathcal{M} /[\mathcal{P}])^{\mathrm{op}}\right)^{\mathrm{op}}$. 
(2) If $\mathcal{C}$ has enough injectives $\mathcal{I}$ and $\mathcal{M}$ contains $\mathcal{I}$, then $\mathcal{M}_{R} /[\mathcal{M}] \cong\left(\bmod -(\mathcal{M} /[\mathcal{I}])^{\mathrm{op}}\right)^{\mathrm{op}}$ and $\mathcal{M}_{R} /[\Sigma \mathcal{M}] \cong \bmod -(\mathcal{M} /[\mathcal{I}])$.

Proof. We only prove (1). It follows from Theorem 4.1 and Lemma 5.3 .

Definition 5.5. ([11, Definition 5.3]) Let $\mathcal{C}$ be an extriangulated category with enough projectives and enough injectives. A full subcategory $\mathcal{M}$ is called $n$-cluster tilting for some integer $n \geq 2$, if it satisfies the following conditions.

(1) $\mathcal{M}$ is functorially finite in $\mathcal{C}$.

(2) $X \in \mathcal{M}$ if and only if $\mathbb{E}^{i}(X, \mathcal{M})=0$ for $i \in\{1,2, \cdots, n-1\}$.

(3) $X \in \mathcal{M}$ if and only if $\mathbb{E}^{i}(\mathcal{M}, X)=0$ for $i \in\{1,2, \cdots, n-1\}$.

In particular, a 2-cluster tilting subcategory of $\mathcal{C}$ is simply called cluster tilting subcategory.

Assume that $\mathcal{M}$ is an $n$-cluster tilting subcategory. Define

$$
{ }^{\perp_{n-2}} \mathcal{M}=\left\{X \in \mathcal{C} \mid \mathbb{E}^{i}(X, \mathcal{M})=0, i \in\{1,2, \cdots, n-2\}\right\}
$$

and $\mathcal{M}^{\perp_{n-2}}=\left\{X \in \mathcal{C} \mid \mathbb{E}^{i}(\mathcal{M}, X)=0, i \in\{1,2, \cdots, n-2\}\right\}$.

Proposition 5.6. Let $\mathcal{M}$ be an $n$-cluster tilting subcategory of an extriangulated category $\mathcal{C}$, then $\mathcal{M}_{L}={ }^{\perp_{n-2}} \mathcal{M}$ and $\mathcal{M}_{R}=\mathcal{M}^{\perp_{n-2}}$.

Proof. We only prove the first equation. Let $X \in^{\perp_{n-2}} \mathcal{M}$ and $X \stackrel{x}{\longrightarrow} I \stackrel{y}{\longrightarrow} C-\stackrel{\delta}{-} \rightarrow$ be an $\mathfrak{s}$-triangle with $I \in \mathcal{I}$. Assume that $f: X \rightarrow M$ is a left $\mathcal{M}$-approximation of $X$. By Lemma 2.8, we have the following morphism of $\mathfrak{s}$-triangles

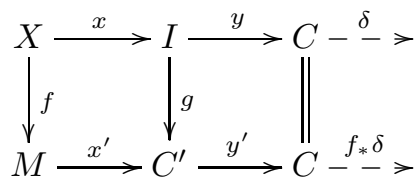

such that $X \stackrel{\left(\begin{array}{l}f \\ x\end{array}\right)}{\longrightarrow} M \oplus \stackrel{\left(x^{\prime},-g\right)}{\longrightarrow} C^{\prime} \stackrel{y^{\prime *} \delta}{-}-$ is an $\mathfrak{s}$-triangle. We claim that $C^{\prime} \in \mathcal{M}$, thus $X \in \mathcal{M}_{L}$. In fact, for each $M^{\prime} \in \mathcal{M}$, by Lemma 2.10 we have the following exact sequence

$\mathcal{C}\left(C^{\prime}, M^{\prime}\right) \rightarrow \mathcal{C}\left(M \oplus I, M^{\prime}\right) \rightarrow \mathcal{C}\left(X, M^{\prime}\right) \rightarrow \mathbb{E}\left(C^{\prime}, M^{\prime}\right) \rightarrow \mathbb{E}\left(M \oplus I, M^{\prime}\right) \rightarrow \mathbb{E}\left(X, M^{\prime}\right)$ $\rightarrow \cdots \rightarrow \mathbb{E}^{i}\left(M \oplus I, M^{\prime}\right) \rightarrow \mathbb{E}^{i}\left(X, M^{\prime}\right) \rightarrow \mathbb{E}^{i+1}\left(C^{\prime}, M^{\prime}\right) \rightarrow \mathbb{E}^{i+1}\left(M \oplus I, M^{\prime}\right) \rightarrow \cdots$. Since $\mathbb{E}^{i}\left(M \oplus I, M^{\prime}\right)=\mathbb{E}^{i+1}\left(M \oplus I, M^{\prime}\right)=0$, we get $\mathbb{E}^{i}\left(X, M^{\prime}\right) \cong \mathbb{E}^{i+1}\left(C^{\prime}, M^{\prime}\right)$ for $i \in\{1,2, \cdots, n-2\}$. Thus, as $X \in^{\perp_{n-2}} \mathcal{M}, \mathbb{E}^{i}\left(C^{\prime}, M^{\prime}\right)=0$ if $2 \leq i \leq n-1$. Noting that $f: X \rightarrow M$ is a left $\mathcal{M}$-approximation, we have $\mathbb{E}\left(C^{\prime}, M^{\prime}\right)=0$. Therefore $C^{\prime} \in \mathcal{M}$.

Suppose that $X \in \mathcal{M}_{L}$. There exists an s-triangle $X \stackrel{f_{1}}{\longrightarrow} M_{1} \stackrel{f_{2}}{\longrightarrow} M_{2}-{ }_{-} \rightarrow$ with $M_{1}, M_{2} \in \mathcal{M}$. For any $M \in \mathcal{M}$, we have an exact sequence

$$
0=\mathbb{E}^{i}\left(M_{1}, M\right) \rightarrow \mathbb{E}^{i}(X, M) \rightarrow \mathbb{E}^{i+1}\left(M_{2}, M\right)=0
$$

for $i \in\{1,2, \cdots, n-2\}$. Therefore, $X \in^{\perp_{n-2}} \mathcal{M}$.

Corollary 5.7. Let $\mathcal{C}$ be an extriangulated category with enough projectives $\mathcal{P}$ and enough injectives $\mathcal{I}$. If $\mathcal{M}$ is a cluster tilting subcategory of $\mathcal{C}$, then

(1) $\mathcal{C} /[\mathcal{M}] \cong \bmod -(\mathcal{M} /[\mathcal{P}]) \cong\left(\bmod -(\mathcal{M} /[\mathcal{I}])^{\mathrm{op}}\right)^{\mathrm{op}}$.

(2) $\mathcal{C} /[\Omega \mathcal{M}] \cong\left(\bmod -(\mathcal{M} /[\mathcal{P}])^{\mathrm{op}}\right)^{\mathrm{op}}$.

(3) $\mathcal{C} /[\Sigma \mathcal{M}] \cong \bmod -(\mathcal{M} /[\mathcal{I}])$. 
Remark 5.8. Let $\mathcal{M}$ be a cluster tilting subcategory of an extriangulated category $\mathcal{C}$. Then the quotient category $\mathcal{C} /[\mathcal{M}]$ is abelian can follows from the theory of cotorsion pairs on extriangulated categories or that of one-sided triangulated categories; see [11, Theorem 3.2] and [5, Theorem 3.3].

\section{REFERENCES}

[1] A. B. Buan, R. Marsh, I. Reiten, Cluster-tilted algebras, Trans. Amer. Math. Soc. 359(1)(2007) 323-332.

[2] L. Demonet, Y. Liu, Quotients of exact categories by cluster tilting subcategories as module categories, J. Pure Appl. Algebra, 217(12)(2013) 2282-2297.

[3] Ö. Eiríksson, From submodule categories to the stable Auslander algebra, J. Algebra, 486(2017) 98-118.

[4] R. Gentle, A study of the sequence category, University of British Columbia, 1982.

[5] J. He, P. Zhou, Abelian quotients of extriangulated categories, Proc. Indian Acad. Sci. Math. Sci. 129 (2019), no. 4, Art. 61, 11 pp.

[6] O. Iyama,H. Nakaoka, Y. Palu, Auslander-Reiten theory in extriangulated categories, arXiv:1805.03776v1,2018.

[7] O. Iyama, Y. Yoshino, Mutation in triangulated categories and rigid Cohen-Macaulay modules, Invent. Math. 172(1)(2008) 117-168.

[8] B. Keller, I. Reiten, Cluster-tilted algebras are Gorenstein and stably Calabi-Yau, Adv. Math. 211(1)(2007) 123-151.

[9] S. Koenig, B. Zhu, From triangulated categories to abelian categories: cluster tilting in a general framework, Math. Z. 258(1)(2008) 143-160.

[10] Z. Lin, Abelian quotients of category of short exact sequeces, J. Algebra 551(2020) 61-92.

[11] Y. Liu, H. Nakaoka, Hearts of twin cotorsion pairs on extriangulated categories, J. Algebra 528 (2019) 96-149.

[12] Y.Liu, P.Zhou, Abelian Categories Arising from Cluster Tilting Subcategories, Appl. Categ. Structures, 28 (2020) 575-594.

[13] Y.Liu, P.Zhou, Abelian categoris arising from cluster-tilting subcategories II: quotient functors, arXiv:1809.06597 2018. To appear in Proceedings of the Royal Society of Edinburgh Section A: Mathematics.

[14] A. Neeman, Triangulated Categories, Annals of Mathematics Studies, vol. 148, Princeton University Press, Princeton, NJ, 2001.

[15] H. Nakaoka, Y. Palu, Extriangulated categories, Hovey twin cotorsion pairs and model structures. Cah. Topol. Géom. Diffr. Catég. 60(2)(2019) 117-193.

[16] C. M. Ringel, P. Zhang, From submodule categories to preprojective algebras, Math. Z. 278(1-2)(2014) 55-73.

[17] P. Zhou, B. Zhu, Triangulated quotient categories revisited, J. Algebra 502 (2018) 196-232.

School of Mathematical sciences, Huaqiao University, Quanzhou 362021, China.

E-mail address: lzq134@163.com 